\title{
Urinary glycoprofile identifies presence and grade of cancer
}

A urinary glycoprofile marker (UGM) is able to discriminate between urine samples from healthy men, men with $\mathrm{BPH}$ and men with newly diagnosed prostate cancer, even when serum PSA values fall within a diagnostic 'grey zone' of $4-10 \mathrm{ng} / \mathrm{ml}$. The urinary glycoprofile also shows significant differences between samples from men with poorly, moderately and well differentiated prostate cancer.

\section{Tumorigenesis is associated with aberrant $\mathrm{N}$-glycosylation of proteins, such as PSA $\$ 7$}

Serum PSA is a well-established, but far from ideal, marker for diagnosis of prostate cancer. In addition to problems with sensitivity and specificity, widespread serum PSA screening has led to substantial overtreatment of low-risk disease. Better markers are urgently required.

A common post-translational modification of proteins is the attachment of oligosaccharides (glycans) to asparagine residues ( $N$-glycosylation). Tumorigenesis is associated with aberrant $N$-glycosylation of proteins, such as PSA. Prostatic stimulation by digital rectal examination (DRE) induces the release of proteins into urine. The glycoprofile of a urine sample, determined by measurement of isolated, fluorescently labelled glycans using a DNA sequencer, is combined with prostate volume to calculate the UGM, which has been shown to add value to serum PSA for diagnosis of $\mathrm{BPH}$ and prostate cancer.

Vermassen and colleagues have now compared glycoprofiles from 54 healthy men, 99 men with BPH (PSA density $<0.15 \mathrm{ng} / \mathrm{ml}^{2}$, normal DRE) and 74 men with prostate cancer (PSA density $>0.151 \mathrm{ng} / \mathrm{ml}^{2}$, abnormal DRE). Prostate cancer stage was determined using multiparametric MRI (mpMRI) and prostate biopsy.

Glycoprofiles were significantly different between healthy men and men with $\mathrm{BPH}$ $(P<0.001)$ or prostate cancer $(P<0.001)$, and between men with $\mathrm{BPH}$ and prostate cancer $(P<0.001)$. In the diagnostic grey zone, serum PSA was not able to discriminate
$\mathrm{BPH}$ from cancer, but this distinction was possible using UGM $(P<0.001)$.

No correlation was observed between serum PSA concentration or prostate volume and cancer stage. By contrast, patterns of fucosylation differed significantly between urine samples from patients with low-grade (Gleason score $<7$ ) and high-grade (Gleason score $>7$ ) tumours $(P=0.01)$, and between intermediategrade (Gleason score $=7$ ) and high-grade tumours $(P=0.011)$. The urinary glycoprofile could not differentiate lowgrade from intermediate-grade tumours, but MRI could.

UGM measurement is noninvasive (discounting the DRE), more accurate than serum PSA and less expensive than mpMRI. The potential clinical utility of UGM should now be thoroughly assessed.

Robert Phillips

Original article Vermassen, T. et al. Urinary prostate protein glycosylation profiling as a diagnostic biomarker for prostate cancer. Prostate doi:10.1002/pros.22918 